Rev. Biol. Trop. 52(1): 229-238, 2004

www.ucr.ac.cr www.ots.ac.cr www.ots.duke.edu

\title{
Ecología reproductiva y cacería de la tortuga Trachemys scripta (Testudinata: Emydidae), en el área de la Depresión Momposina, norte de Colombia
}

\author{
Marcela Bernal Múnera ${ }^{1}$, Juan Manuel Daza R. ${ }^{2}$ \& Vivian P. Páez ${ }^{3}$ \\ Fundación Neotrópicos, Calle 50 N 38-50. Medellín, Colombia; marcelabernal@hotmail.com \\ Corporación Ambiental, Universidad de Antioquia. Medellín, Colombia; juandaza35@hotmail.com \\ Instituto de Biología, Universidad de Antioquia. Medellín, Colombia. Fax: (574)2330120; vpaez@quimbaya.udea.edu.co
}

Recibido 05-VII-2001. C Corregido 21-I-2002. $\quad$ Aceptado 19-IV-2002.

\begin{abstract}
We studied the reproductive ecology of the slider turtle, Trachemys scripta callirostris, during the reproductive season of the year 2000, in an area of the Mompós Depression, northern Colombia. We examined the number of nests ovoposited in locations with different characteristics, their hatching success rates and the natural causes of egg mortality. We also determined the effects of exploitation of nesting females, in two populations with different levels of harvesting. The results showed that the majority of nests were laid along bodies of water, in the first $20 \mathrm{~m}$ of the shoreline, in sites with substrates of moderate humidity, sand and clayish texture, covered by creeping vegetation. Invertebrates produced the greatest mortality in the nests. Hatching success was high, compared to other reports for this species. Only in the site with the highest hunting pressure, a negative correlation was found, between the harvest rate and the time elapsed since the start of the nesting period, suggesting that the excessive extraction decreased the number of nesting females. Also, the females from the highly exploited site were significantly smaller than those from the less exploited site. The females, neonates and nests of the entire area, exhibited sizes below that reported for other tropical populations of the genus. The continued hunting pressure in northern Colombia, appears to be a factor that has altered the structure and population dynamics of $T$. scripta callirostris.
\end{abstract}

Key words: Testudinata, Emydidae, Trachemys scripta callirostris, nesting, reproductive success, hunting, Colombia.

La tortuga icotea, Trachemys scripta (Schoepf 1792), tiene el ámbito de distribución más extenso en comparación a cualquier otra tortuga acuática en el mundo, extendiéndose desde el sur de Michigan (EEUU), hasta el norte de Argentina (Moll y Legler 1971). Es probablemente la tortuga más conocida en el mundo, ya que debido a su popularidad como mascota en Estados Unidos y Europa, se convirtió en sujeto de numerosos estudios fisiológicos, ecológicos y de comportamiento (Carr 1952, Moll y Legler 1971, Acuña et al. 1983, Acuña-Mesén 1989, Ernst y Barbour 1989, Gibbons 1990).

La mortalidad natural que soporta esta especie es muy alta, especialmente sobre los hue- vos y los neonatos, ya que estos estadíos son más susceptibles a la depredación (Moll y Legler 1971, Medem 1975) y la mayoría de los animales carnívoros y omnívoros en las áreas de anidación, comen huevos de esta tortuga (Moll y Legler 1971, Moll 1986, Fachin 1994). Además de la depredación por vertebrados, algunos invertebrados (hormigas y moscas) también atacan huevos de T. scripta. Estos factores, unidos a la pérdida por inundación, incrementan el porcentaje de mortalidad hasta casi el $100 \%$, en algunos sitios de anidación (Moll y Legler 1971).

La subespecie Trachemys scripta callirostris (Gray 1885), es endémica de Colombia 
(Medem 1975) y se distribuye en la cuenca del río Magdalena y a lo largo de la costa Atlántica (Castaño-Mora 1997). El único estudio ecológico publicado sobre $T$. scripta callirostris en Colombia, fue hecho por Medem (1975) y en él se estudiaron algunos parámetros de la ecología reproductiva.

Actualmente T. scripta callirostris es una de las tortugas más explotadas en Colombia (Castaño-Mora 1997), por lo que se encuentra listada en el Apéndice II del CITES (Groombridge 1982). La principal razón de la importancia económica de las icoteas, radica en su uso como fuente de proteína fácilmente cosechable; la carne, el hígado, los huevos y los folículos ováricos son altamente apreciados por su buen sabor (Moll y Legler 1971, Medem 1975), además los tortuguillos son vendidos en grandes cantidades como mascotas, en las principales ciudades de Colombia (CastañoMora 1997). En varias regiones de Colombia las poblaciones de esta subespecie han sido prácticamente extirpadas debido a la sobrexplotación y en otras, el número de individuos se ha visto disminuido considerablemente (Medem 1975).

El objetivo del presente estudio fue determinar algunos aspectos de la ecología reproductiva de la subespecie tales como, tamaño de nidadas y de neonatos, éxito de eclosión, causas de mortalidad natural de los huevos y número de nidos en áreas con características diferentes; también, se pretendió evaluar el efecto que tiene la cacería sobre las poblaciones de hembras anidantes de icotea, en sitios altamente intervenidos por el hombre.

\section{MATERIALES Y MÉTODOS}

El estudio fue llevado a cabo de febrero a mayo del 2000, en el área de la Depresión Momposina, al norte de Colombia, en dos sitios que se encuentran alejados entre sí por $100 \mathrm{~km}$, aproximadamente.

El primero es el complejo cenagoso de $\mathrm{Pi}$ jiño ( $9^{\circ} 17^{\prime} \mathrm{N}$ y $\left.74^{\circ} 24^{\prime} \mathrm{W}\right)$, en el departamento del Magdalena, el estudio allí se llevó a cabo en la ciénaga Juan Criollo, ubicada al sur del complejo cenagoso y en la Isla León, que se encuentra en esta ciénaga. En esta zona, la presión de caza sobre esta tortuga es muy alta, debido a la gran cantidad de asentamientos humanos en las orillas de las ciénagas, al fácil acceso a los sitios donde salen las hembras a anidar y a la carencia de control y restricción por parte de las autoridades. Las ciénagas están interconectadas y aunque el nivel del agua varía en época seca y en época de lluvias, se les considera cuerpos de agua permanentes.

El segundo sitio es el municipio de Hatillo de Loba ( $9^{\circ} 00^{\prime} \mathrm{N}$ y $\left.74^{\circ} 07^{\prime} \mathrm{W}\right)$, en el departamento de Bolívar, donde se escogieron dos lugares, Caño Mocho y la Ciénaga de Jobá, los cuales son cuerpos de agua semipermanentes, que dependen de la creciente del río Magdalena, que surte los pequeños caños que los comunican con éste. En este sitio, la presión de caza es menor en comparación con el complejo cenagoso, debido a que se presentan pocos asentamientos humanos cerca de los cuerpos de agua, el acceso a las orillas es difícil (terreno pantanoso) y por lo tanto no se puede llegar a algunas partes de las ciénagas donde potencialmente pueden anidar las tortugas.

Para documentar las características de los sitios en los que anida esta tortuga, se localizaron 55 nidos en la Isla León y en los alrededores de la ciénaga Juan Criollo, con la ayuda de una persona de la zona, quien se guiaba por la perturbación producida por la tortuga cuando excavaba. Para cada nido se registraron las siguientes características: 1) Distancia total desde el espejo de agua, que recorre la tortuga para depositar su nidada; 2) Tipo de sustrato, de acuerdo a dos variables: humedad, la cual se clasificó cualitativamente de 1 a 4 , siendo 1 el sustrato más seco y 4 el más húmedo, y la textura, que se clasificó cualitativamente en tres clases: limoso, el cual es duro, de apariencia arcillosa y con poca capacidad de drenaje; arenoso-limoso que es más suelto y con mayor capacidad de drenaje y por último, arenoso, que es blando, suelto y con excesiva capacidad de drenaje. 3) Cobertura vegetal del sitio de anidación. 
Se determinó el éxito de eclosión y el tamaño de las nidadas de Isla León, así como el porcentaje de huevos eclosionados con respecto al total de huevos ovopositados. A los neonatos obtenidos de los nidos eclosionados en Isla León, se les midió usando un calibrador de $0.1 \mathrm{~mm}$ de precisión: el largo curvo del caparazón (LCC); ancho curvo del caparazón (ACC) y la longitud del plastrón (LP). Adicionalmente, se determinó su peso con $0.1 \mathrm{~g}$ de precisión.

Para identificar las posibles causas de mortalidad de los huevos, se revisaron los nidos periódicamente durante el periodo de incubación; se registró la presencia de cáscaras fuera del nido, despedazadas y/o con huellas de dientes y los huevos que no eclosionaron después de 70 días, fueron examinados para determinar otras posibles causas de mortalidad. Los invertebrados observados en los huevos o en la cámara, fueron colectados y almacenados en alcohol al 70\%, para luego ser identificados en el laboratorio.

Durante los meses de febrero a marzo del 2000, se estimaron los niveles de cacería en dos sitios (Caño Mocho y la Ciénaga de Jobá), en el municipio de Hatillo de Loba, acompañando a un cazador, quien recorría los alrededores de los cuerpos de agua buscando icoteas, con la ayuda de perros adiestrados. Se calculó la tasa de cacería, como el número de hembras capturadas por área recorrida y el tiempo de esfuerzo empleado por el cazador (tortugas/ha/hr). Con el fin de evidenciar el efecto de la cacería sobre la talla de las hembras anidantes, en dos sitios con diferentes presiones de caza (Pijiño y Hatillo de Loba), se registraron medidas morfométricas de LCC. Los individuos utilizados para este análisis provenían tanto de animales capturados durante las faenas de caza, las cuales se realizaban durante la noche, como de caparazones obtenidos recientemente, que fueron encontrados en las viviendas de los pobladores. Se comprobó que todos estos caparazones provenían de hembras, pues los cazadores las encontraron ovopositando o con huevos en los oviductos.

\section{RESULTADOS}

Características de las áreas de anidación: El rango de distancia a la cual se encontraron los 55 nidos en el área de estudio, varió desde 2 hasta $25 \mathrm{~m}$ y para el análisis, agrupamos los datos en cinco clases de distancias (intervalos de $5 \mathrm{~m}$ ). Aunque el número de nidos encontrados después de los 20 metros desde el cuerpo de agua, es relativamente bajo, no encontramos diferencias significativas en cuanto a la cantidad presente en las cinco clases de distancias $\left(\chi^{2}=7.64, \mathrm{gl}=3, \mathrm{p}>0.05\right)$ (Fig. 1). Se comparó la última clase (20-25 m) con el resto y se encontraron diferencias significativas en el número de nidos hallados en esta clase, con respecto a las otras cuatro $\left(\chi^{2}=5.57, \mathrm{gl}=1\right.$, $\mathrm{p}<0.05)$.

De los 55 nidos evaluados, el 50.9\% (28) se encontraron en substratos moderadamente húmedos (clase 3), con textura limoso-arenosa y su diferencia fue significativa con respecto a las otras clases de sustrato $\left(\chi^{2}=49.86, \mathrm{gl}=5\right.$, p<0.001) (Fig. 2). Sin embargo, al eliminar esta clase del análisis, no encontramos diferencias significativas entre la cantidad de nidos ovopositados en las otras cinco clases de sustrato $\left(\chi^{2}=6.14, \mathrm{gl}=4, \mathrm{p}>0.05\right)$. Además se encontró que existe una asociación significativa entre la textura y la humedad $\left(\chi^{2}=25.51, \mathrm{gl}=6\right.$, $\mathrm{p}<0.001)$.

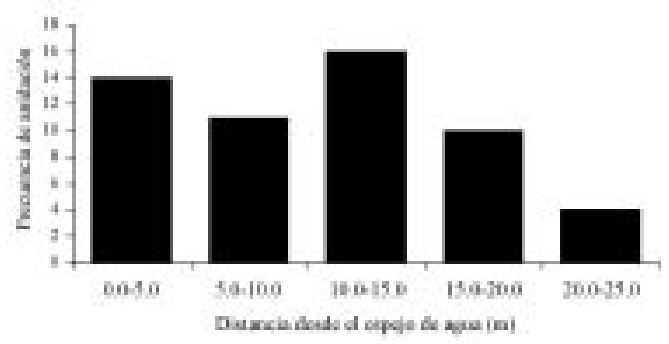

Fig. 1. Preferencia del sitio de postura de Trachemys scripta callirostris con relación a la distancia desde el espejo de agua.

Fig. 1. Preferences for nesting sites in Trachemys scripta callirostris in relation to the distance from the water's edge. 


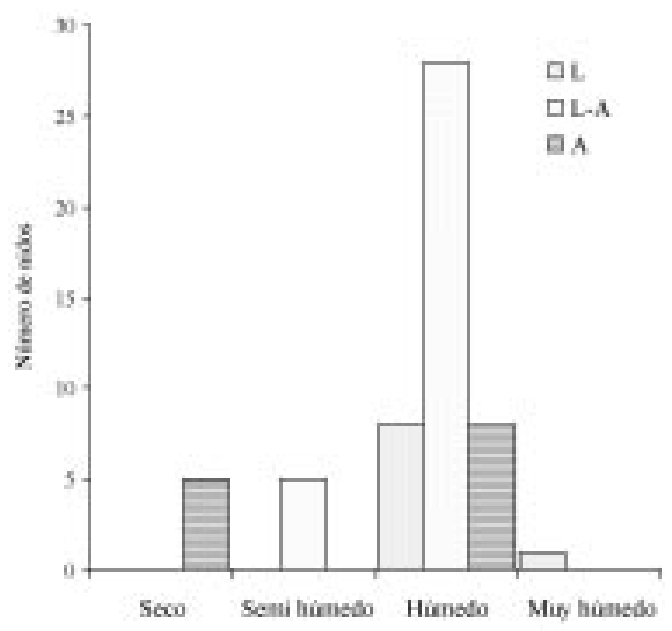

Fig. 2. Preferencia del sitio de anidación de Trachemys scripta callirostris con relación al tipo de sustrato (humedad y textura).

Fig. 2. Preferences for nesting sites in Trachemys scripta callirostris in relation to the type of substrate (humidity and texture).

El 82\% (45) de los nidos examinados estaban cubiertos por plantas acuáticas secas ( $E i$ chornia spp.) o por pasto, diferenciándose significativamente de los nidos bajo otros tipos de coberturas $\left(\chi^{2}=22.45, \mathrm{gl}=3 \mathrm{p}<0.001\right)$ (Fig. $3)$. Aunque existe una gran porción de zonas descubiertas o con arbustos, observamos que la mayoría se encontraba debajo de algún tipo de vegetación rastrera.

Éxito de eclosión y mortalidad de nidos: En la Isla León contabilizamos 247 huevos en 32 nidos, para un promedio de $7.7 \pm 0.57(\mathrm{X} \pm$ $\left.\mathrm{IC}_{(95 \%)}\right)$ huevos por nido, las nidadas estaban constituidas por un mínimo de 2 y un máximo de 15 huevos. De los nidos eclosionados en la isla, obtuvimos 79 neonatos, incluyendo individuos encontrados en nidos que no fueron monitoreados. El LCC fue de $31.9 \pm 0.63 \mathrm{~mm}$, ACC de $29.8 \pm 0.92 \mathrm{~mm}, \mathrm{LP}$ de $29.0 \pm 0.38$ $\mathrm{mm}$ y el peso de $5.6 \pm 0.20 \mathrm{~g}$.

El número de huevos eclosionados (33) fue mucho menor con relación a aquellos que no lo hicieron (214), $\quad\left(\chi^{2}=132.6, \mathrm{gl}=1\right.$, $\mathrm{p}<0.001)$, presentando un éxito de eclosión del $13.4 \%$. El $32 \%$ del total de huevos (79), fueron atacados por invertebrados, entre ellos, hime-

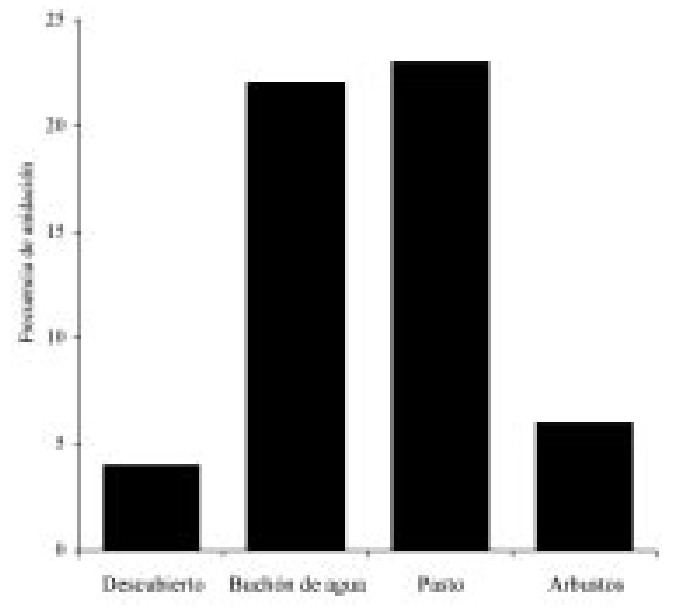

Fig. 3. Preferencia del sitio de anidación de Trachemys scripta callirostris con relación a la cobertura vegetal del sustrato.

Fig. 3. Preferences for nesting sites in Trachemys scripta callirostris in the Jobá marsh (Hatillo de Loba) during the 2000 reproductive season.

nópteros del género Solenopsis, coleópteros del género Canthon y larvas de mosca del género Dohrniphora, éstos consumieron parcial o totalmente el contenido de los huevos. El $23.5 \%$ (58) fueron depredados por algún vertebrado, asociamos esta pérdida principalmente, al lagarto llamado "lobo pollero" (Tupinambis teguixin), ya que observamos varios individuos de esta especie en la Isla León y cerca de los nidos; además las cáscaras de los huevos se encontraban despedazadas y con huellas de dientes, similares a las dejadas en algunos frutos por este lagarto de hábitos omnívoros (Ávila-Pires 1995).

Los nidos que no eclosionaron después de 70 días tenían huevos que habían perdido su turgencia y la cáscara presentaba un color blanco, más opaco del observado en huevos con desarrollo normal. Al disectarlos, encontramos que el $16.6 \%$ de los huevos (41), contenían embriones muertos en diferentes estadios de desarrollo. El $3.6 \%$ de los huevos (9) mostraban señales de descomposición, éstos se encontraban en nidos que se hallaban en zonas anegadas. Las causas de mortalidad mencionadas anteriormente, presentaron diferencias 
significativas en cuanto al número de huevos afectados por cada una de ellas, siendo mayor el porcentaje de huevos perdidos como resultado de la depredación por invertebrados $\left(\chi^{2}=56.1, \mathrm{gl}=3, \mathrm{p}<0.001\right)$. El $10.9 \%$ de los huevos (27) no presentaron desarrollo embrionario.

Cacería: Se realizaron 10 muestreos a lo largo de la temporada de anidación en la Ciénaga de Jobá, que cuenta con una extensión de 40 hectáreas y 6 muestreos en Caño Mocho, con una extensión de 50 hectáreas.

Las tasas de cacería variaron desde 0.17 hasta 1.2 tortugas $/ \mathrm{ha} / \mathrm{hr}$. El análisis de las tasas a lo largo de la temporada en la Ciénaga de Jobá, mostró una correlación negativa estadísticamente significativa $(\mathrm{r}=-0.8, \mathrm{p}<0.01)$ (Fig. 4), a diferencia de Caño Mocho, en donde no se encontró ninguna correlación $(\mathrm{r}=0.06, \mathrm{p}>0.1)$ (Fig. 5).

Examinamos un total de 126 hembras cazadas, entre individuos vivos y caparazones. En el complejo cenagoso de Pijiño (sitio con alta presión de caza) registramos el LCC de 81 hembras, con tamaños que variaron entre 17.0 y $27.9 \mathrm{~cm}$ y con un promedio de $21.7 \pm 0.46$ $\mathrm{cm}$. En la localidad de Hatillo de Loba (sitio con baja presión de caza) medimos 45 individuos con LCC entre 23.7 y $30.0 \mathrm{~cm}$, con un promedio de $26.7 \pm 0.44 \mathrm{~cm}$. Por último, se encontraron diferencias significativas en el tamaño de las hembras cazadas en los dos sitios, siendo menores las de la localidad de Pijiño $(\mathrm{t}=15.2, \mathrm{p}<0.001)$.

\section{DISCUSIÓN}

La cantidad de nidos de T. scripta callirostris hallados en el complejo cenagoso de Pijiño, depende de las características del sitio: se encontró un umbral de $6 \mathrm{~m}$ de distancia en la zona seca útil, por encima de la cual no se halló ningún nido; es posible que a distancias más grandes las hembras se expongan a un mayor número de depredadores (Temple 1987). Las hembras en esta zona no se alejan mucho del agua, inclusive en la zona pantanosa, la cual es amplia, no encontramos nidos a más de

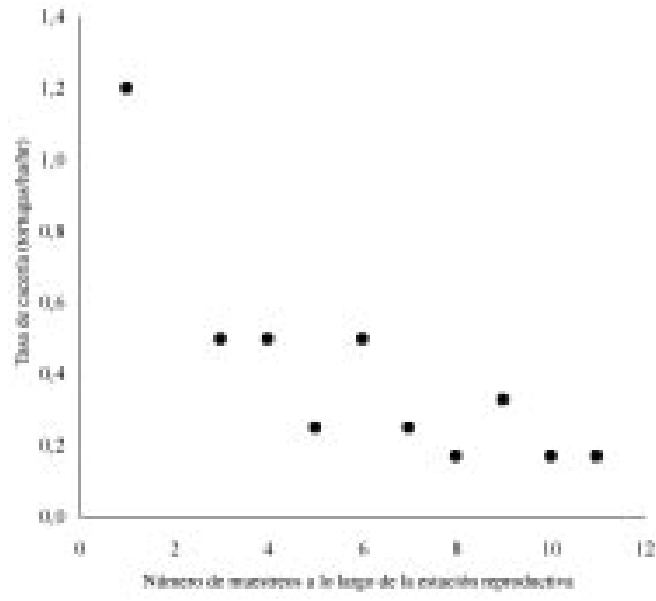

Fig. 4. Tasa de cacería de hembras adultas de Trachemys scripta callirostris en la Ciénaga de Jobá (Hatillo de Loba) durante la temporada reproductiva del año 2000.

Fig. 4. Hunting rate for adult female Trachemys scripta callirostris in the Jobá marsh (Hatillo de Loba) during the 2000 reproductive season.

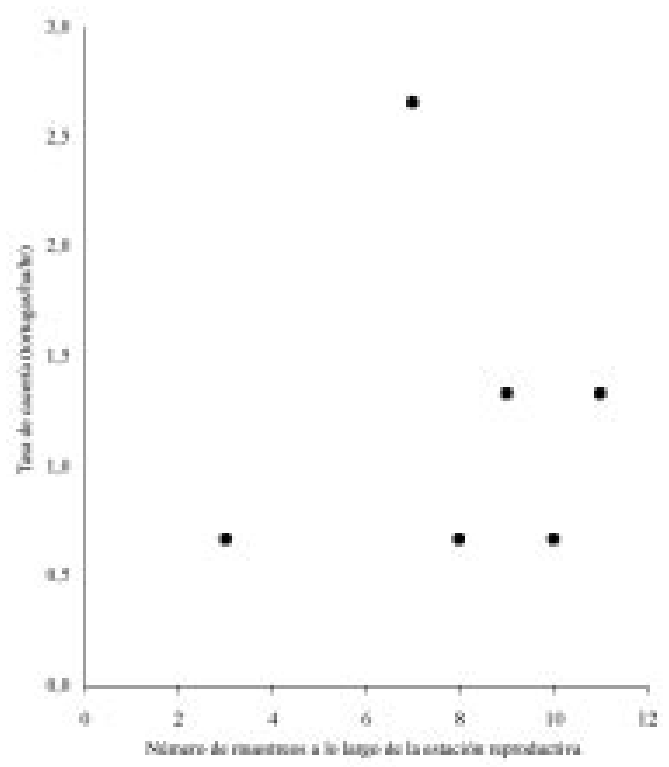

Fig. 5. Tasa de cacería de hembras adultas de Trachemys scripta callirostris en Caño Mocho (Hatillo de Loba) durante la temporada reproductiva del año 2000.

Fig. 5. Hunting rate of adult female Trachemys scripta callirostris in the Mocho channel (Hatillo de Loba) during the 2000 reproductive season. 
$20 \mathrm{~m}$ de la orilla de la ciénaga. Sin embargo, Moll y Legler (1971) informan que la distancia a la cual pueden desplazarse las hembras es muy variable dentro de la especie, ya que en otras poblaciones las hembras parecen desplazarse más en tierra. Estos autores también informan que en Juan Mina (Panamá), la distancia promedio de los nidos de $T$. scripta al agua fue de $50 \mathrm{~m}$, con un rango de 2 a 320 m. Medem (1975) encontró que en la Ciénaga del Totumo (Colombia), los nidos se encontraban a una distancia entre 2 a $52 \mathrm{~m}$ de la orilla. Esta diferencia puede deberse, a que la tasa de cacería en nuestra área de estudio es mucho mayor, lo que implica que a mayor desplazamiento de la tortuga, menor es la distancia que separa al nido de los asentamientos humanos de la zona, y por lo tanto es más alta la probabilidad de que las hembras sean capturadas o el nido saqueado, en comparación con aquellas que anidan cerca de las orillas.

Los resultados muestran un mayor número de nidos ovopositados en sitios con humedad moderada (clase 3) y textura limoso-arenosa, en donde los huevos pueden absorber agua e incrementar su masa al inicio del periodo de incubación (Morris et al. 1983, Garduño y Cervantes 1996), lo cual puede influir en que los neonatos sean de mayor tamaño y por lo tanto, cuenten con mayores posibilidades de supervivencia (Cagle et al. 1993).

Por otra parte, los incubados en sustratos relativamente secos pueden perder agua y de este modo, pierden masa continuamente durante el periodo de desarrollo (Morris et al. 1983, Garduño y Cervantes 1996). Sin embargo, lugares demasiado húmedos también pueden incrementar las infecciones de la nidada por bacterias y hongos, y en caso de inundación, se puede perder el nido (Garduño y Cervantes 1996).

Se observó una tendencia de los sustratos arenosos a ser más secos y de los limosos a ser más húmedos, esto puede atribuirse a la asociación hallada entre la humedad y la textura, ya que ésta última determina la capacidad de drenaje del suelo. Congdon y Gibbons (1985) informaron que las nidadas de las poblaciones de T. scripta en Norteamérica, usualmente son construidas en suelos con buen drenaje, esto es fundamental para el desarrollo de los embriones, ya que en los nidos puestos en suelos muy arcillosos, se restringe el intercambio gaseoso entre la cámara y el suelo que la rodea (Congdon y Gibbons 1990). Estas diferencias pueden presentarse debido a que existe una mayor disponibilidad del sustrato limoso-arenoso con humedad moderada en nuestra área de estudio, es decir, que la mayor parte del área efectiva para la anidación, presenta estas características. Es importante anotar que la temperatura, la humedad y la textura del suelo son críticas para la tasa de desarrollo, la supervivencia del embrión y la determinación sexual (Congdon y Gibbons 1990).

La mayoría de los nidos fueron encontrados bajo vegetación rastrera, plantas acuáticas y pasto, lo que posiblemente disminuye la detección por parte de los depredadores, siendo ventajoso para su supervivencia. La vegetación inmediatamente encima del nido, puede mantener más constante la temperatura de éste, a diferencia de sitios totalmente descubiertos, donde las temperaturas fluctúan de manera drástica a lo largo del día. Sin embargo, esta condición contribuye a reducir un poco la temperatura interna del nido, lo que podría afectar la proporción de sexos, ya que en esta especie el sexo lo determina la temperatura (Gibbons 1990).

El factor más importante para la pérdida de huevos fue la infestación por invertebrados, resultado que concuerda con estudios previos en tortugas (Moll y Legler 1971, Medem 1975, Fachin 1994). No fue posible determinar si todos los invertebrados encontrados en los huevos son depredadores o consumidores oportunistas, sin embargo, en el caso de las hormigas de la familia Formicidae y de las larvas de la familia Phoridae, estas ya han sido informadas como depredadoras de huevos de tortuga (Carpenter 1960, Moll y Legler 1971, Acuña-Mesén y Hanson 1990, Fachin 1994).

Aunque la literatura informa varios vertebrados que son depredadores naturales de las nidadas de icotea, atribuimos la pérdida de nidadas por vertebrados al lobo pollero (T. teguixin), por 
ser el único vertebrado observado en la isla y cerca de los nidos. Debe tenerse en cuenta que algunas aves y mamíferos nocturnos pudieron haber depredado algunos nidos sin que fueran detectados.

El mayor éxito de eclosión en comparación con otras poblaciones o especies del mismo género, puede deberse a que las nidadas monitoreadas se encontraban en una isla, lo que posiblemente afectó las tasas de mortalidad por vertebrados, debido a que la presencia de éstos es menor en islas que en áreas continentales (Rand y Robinson 1969). También es importante destacar que esta zona ha sido bastante perturbada, lo que ha disminuido considerablemente la fauna silvestre presente en ella (Cuadro 1).

Es fundamental que en el momento de determinar los daños sobre una población, se tenga en cuenta que la depredación por vertebrados, en la mayoría de los casos, conlleva a una pérdida total de la nidada, mientras que los invertebrados pueden atacar solo una parte de ella.

La disminución de la tasa de cacería a lo largo del período de anidación en el sitio de mayor explotación (Ciénaga de Jobá), puede deberse a que una gran cantidad de hembras son removidas al inicio de la temporada y por lo tanto la disponibilidad de este recurso al final de la estación es menor. Por otra parte en Caño Mocho, no se encontró esta disminución, posiblemente porque tiene una menor presión de explotación, ya que está más alejado que la Ciénaga de Jobá, de los asentamientos humanos y presenta una dificultad mayor para el ac- ceso al cuerpo de agua, debido a que sus orillas son bastante pantanosas. Se ha observado que las poblaciones de animales que se encuentran en un hábitat donde pueden ser fácilmente cazadas, están más sujetas al agotamiento que aquellas que se encuentran en áreas de difícil acceso (Moll 1986).

En muchos estudios con tortugas semiacuáticas, se realizan censos por avistamiento de los animales mientras realizan diferentes actividades (principalmente asoleándose), pero en nuestro estudio esta clase de censo no pudo desarrollarse, debido a que nunca observamos tortugas expuestas, ni en los cuerpos de agua, ni desplazándose en tierra, para actividades diferentes a la anidación. En sitios como Coosa River (EEUU), se informa una tortuga por cada $3.3 \mathrm{~m}$ y en el río Chagres (Panamá), una tortuga por cada $6 \mathrm{~m}$ de río (Moll y Legler 1971); a pesar de que estos resultados se obtuvieron usando otra metodología, este número de individuos en comparación a los 0.17-1.2 individuos por hectárea encontrados en la Depresión Momposina, nos muestra un panorama bastante desolador.

En el complejo cenagoso de Pijiño la presión de caza es mayor por la gran cantidad de asentamientos humanos cerca de los cuerpos de agua y debido a que el acceso a las orillas es más fácil ya que posee una mayor elevación y un terreno menos pantanoso. La diferencia encontrada entre el tamaño de las hembras anidantes en Pijiño y Hatillo de Loba, puede ser la consecuencia de una mayor sobreexplotación, en donde se han ido cazando a lo largo del tiempo, las hembras maduras con mayor potencial

CUADRO 1

Éxito de eclosión en varias poblaciones naturales de Trachemys spp. en su rango de distribución

TABLE 1

Hatching success in several natural populations of Trachemys spp. throughout its range of distribution

$\begin{array}{llcl}\text { Especie } & \text { Localidad } & \text { Éxito de eclosión } & \text { Referencia } \\ & & & \\ \text { T. scripta callirostris } & \text { Isla León (Colombia) } & 13.4 \% & \text { Presente estudio } \\ \text { T. scripta } & \text { Juan Mina (Panamá) } & 3 \% & \text { Moll y Legler 1971 } \\ \text { T. scripta } & \text { Louisiana (EEUU) } & 2 \% & \text { Cagle y Chaney 1950 } \\ \text { T. floridana peninsularis } & \text { Florida (EEUU) } & 5 \% & \text { En Gibbons 1990 }\end{array}$


reproductivo y las que se capturan ahora son hembras primerizas de menor tamaño.

Aunque las comparaciones entre poblaciones de tan amplia distribución no son siempre apropiadas, al comparar el tamaño de las hembras de Hatillo de Loba, la población de mayor tamaño en nuestro estudio, con el de otras poblaciones tropicales de la misma especie, encontramos que aparentemente sólo son de mayor tamaño que las de Trachemys scripta chichiriviche en Venezuela, país en donde estas poblaciones también son explotadas (Cuadro 2). En el estudio de Moll y Legler (1971) en Panamá, sitio en el cual prácticamente no hay cacería, las hembras son de mayor tamaño a las de nuestro estudio, apoyando la hipótesis de que aunque en algunos sitios de la Depresión Momposina se presenta más cacería que en otros, es una zona en donde, como informa Medem (1975), esta actividad viene llevándose a cabo desde hace varias décadas, posiblemente disminuyendo el tamaño de las hembras reproductivas. Otros autores también han informado que el promedio de la talla de los adultos puede disminuir por la sobrexplotación para consumo humano (Medem 1975, Pritchard y Trebbau 1984)

El tamaño de las nidadas y de los neonatos de Isla León son menores en tamaño y/o peso en comparación con otras poblaciones de la misma especie en otros sitios (Cuadros $3 \mathrm{y}$ 4 ). Algunos estudios informan que el tamaño de la nidada y/o el tamaño del neonato y del huevo incrementan con el tamaño corporal de la hembra (Caldwell 1959, Carpenter 1960, Semlitsch y Gibbons 1978, Ewert 1979, Stewart 1979, Dunham et al. 1988, Wilbur y Morin 1988).

CUADRO 2

Características morfométricas de hembras adultas en varias poblaciones de Trachemys scripta

TABLE 2

Morphometric characteristics of adult females from several populations of Trachemys scripta

\begin{tabular}{|c|c|c|c|c|}
\hline Especie & Localidad & $\operatorname{LCC}(\mathrm{cm})$ & Rango (cm) & Referencia \\
\hline T. scripta callirostris & Pijiño (Colombia) & 21.7 & $17-27.9$ & Presente estudio \\
\hline T. scripta callirostris & Hatillo de Loba (Colombia) & 26.7 & $23.7-30.0$ & Presente estudio \\
\hline T. scripta callirostris & Cienaga Totumo, (Colombia) & 30.0 & & Medem 1975 \\
\hline T. scripta & Juan Mina (Panamá) & 35.2 & & Moll y Legler 1971 \\
\hline T. scripta chichiriviche & Venezuela & $19.5-32.5$ & & Gibbons 1990 \\
\hline
\end{tabular}

CUADRO 3

Características morfométricas de neonatos en varias poblaciones de Trachemys scripta

TABLE 3

Morphometric characteristics of hatchlings from several populations of Trachemys scripta

$\begin{array}{ccccccc}\text { Especie } & \mathrm{n} & \text { LCC } & \text { ACC } & \text { LP } & \text { Peso } & \text { Referencia } \\ \begin{array}{c}\text { T. scripta callirostris } \\ \text { Isla León (Colombia) }\end{array} & 79 & 31.9 \pm 0.63 & 29.8 \pm 0.92 & 29.0 \pm 0.38 & 5.6 \pm 0.20 & \text { Presente estudio } \\ \text { Cartagena (Colombia) } & 42 & 29.5-39 & & & 7.6-13.3 & \text { Medem 1975 } \\ \begin{array}{c}\text { T. scripta } \\ \text { Juan Mina (Panamá) }\end{array} & 42 & 36.5 & & & \\ \begin{array}{c}\text { T. scripta venusta } \\ \text { México }\end{array} & 154 & 31.8 \pm 0.05 & & & \text { Moll y Legler } 1971 \\ \end{array}$


CUADRO 4

Tamaño de nidadas en varias poblaciones naturales de Trachemys scripta

TABLE 4

Clutch size in several natural populations of Trachemys scripta

\begin{tabular}{|c|c|c|c|c|}
\hline Especie & Localidad & $\left(\mathrm{X} \pm \mathrm{IC}_{(95 \%)}\right)$ & Rango & Referencia \\
\hline T. scripta callirostris & Isla León (Colombia) & $7.7 \pm 0.57$ & $2-15$ & Presente estudio \\
\hline T. scripta callirostris & Chocó (Colombia) & & $12-24$ & Medem 1962 \\
\hline T. scripta callirostris & Cartagena (Colombia) & & $9-25$ & Medem 1975 \\
\hline T. scripta chichiriviche & Venezuela & & $11-28$ & En Gibbons 1990 \\
\hline T. scripta & Juan Mina (Panamá) & 17 & $9-25$ & Moll y Legler 1971 \\
\hline
\end{tabular}

Finalmente, características tales como tamaño de nidada, morfometría de neonatos y de hembras anidantes de las poblaciones de $T$. scripta callirostris del norte de Colombia, parecen reflejar las consecuencias de la cacería excesiva y prolongada de la que han sido objeto durante varias décadas. Es evidente que el estudio de las poblaciones en sitios donde existe una fuerte presión por parte del hombre, puede demostrar los efectos negativos sobre la dinámica poblacional y la ecología reproductiva, que se manifiestan después de una sobrexplotación constante durante largo tiempo.

\section{AGRADECIMIENTOS}

Al Fondo por Estampilla de la Universidad de Antioquia, por su apoyo económico y a la Corporación Ambiental de la Universidad de Antioquia por su ayuda administrativa. A la Fundación Neotrópicos, por su apoyo logístico. A A.M. Ortega, J.J. Estrada y E.M. Muñoz, por su ayuda en la toma de datos y a B.C. Bock por su asesoría y sugerencias al manuscrito. A los habitantes de la zona, por su hospitalidad y enorme colaboración.

\section{RESUMEN}

Se estudió la ecología reproductiva de la tortuga icotea, Trachemys scripta callirostris, durante la estación reproductiva del 2000, en una localidad de la Depresión Momposina, al norte de Colombia. Se examinó el número de nidos en áreas con características diferentes, el éxito de eclosión y las causas de mortalidad natural de los huevos. Se determinó el efecto de la explotación sobre las poblaciones de hembras anidantes en dos localidades con diferentes tasas de cacería. Los resultados mostraron que la mayor cantidad de nidos se encontraron entre los primeros $20 \mathrm{~m}$, a partir de la orilla de los cuerpos de agua, en sitios con sustratos de humedad moderada y textura limoso-arenosa, cubiertos con vegetación rastrera. Los invertebrados produjeron la mayor mortalidad en las nidadas. El éxito de eclosión de la población estudiada fue mayor en comparación con otros informes de la misma especie. En el sitio que presenta mayor presión de caza se encontró una correlación negativa entre la tasa de cacería y los días transcurridos desde el inicio de la temporada de anidación, lo que probablemente se deba a que la extracción excesiva disminuye la cantidad de hembras anidantes hacia el fin de la temporada. Las hembras del sitio con mayor explotación, presentaron una talla significativamente menor con relación a las del sitio con menor caza. Las hembras, neonatos y nidadas de toda la zona, mostraron menor tamaño que las de otras poblaciones tropicales del mismo género. La prolongada presión de caza en el norte de Colombia parece ser un factor de alteración de la estructura y la dinámica poblacional de T. scripta callirostris.

\section{REFERENCIAS}

Acuña, R., A. Casting \& F. Flores. 1983. Aspectos ecológicos de la distribución de las tortugas terrestres y semiacuáticas en el Valle Central de Costa Rica. Rev. Biol. Trop. 31: 181-192.

Acuña-Mesén, R.A. 1989. Ultraestructura de la cáscara del huevo de la tortuga Pseudemys scripta (Testudines: Emydidae). Rev. Biol. Trop. 37: 193-200.

Acuña-Mesén, R. \& P.E. Hanson. 1990. Phorid fly larvae as predators of turtle eggs. Herp. Rev. 21(1): 13-14.

Avila-Pires, T. 1995. Lizards of Brazilian Amazonia. Zool. Verh. Leiden 299: 1-706. 
Cagle, F.R. \& Chaney. 1950. Turtle populations in Louisiana. Amer. Midl. Nat. 43: 383-388.

Cagle, K.D., G.C. Packard, K. Miller \& M.J. Packard. 1993. Effects of the microclimate in natural nests on development of embryonic painted turtles, Chrysemys picta. Function. Ecol. 7: 653-660.

Caldwell, D.K. 1959. The loggerhead turtles of Cape Romain, South Carolina. Bull. Fla. State Mus. Biol. Sci. 4: 319-348.

Carpenter, C.C. 1960. Reproduction in Oklahoma Sceloporus and Cnemidophorus. Herpetologica 11: 120.

Carr, A. 1952. Handbook of turtles: the turtles of the United States, Canada and Baja California. Comstock. Ithaca, Nueva York. 542 p.

Castaño-Mora, O.V. 1997. Status of the tortoises and freshwater turtles of Colombia, pp. 302-306. In J. Van Abbema (ed.). Proceedings: Conservation, Restoration and Management of Tortoises and Turtles An International Conference. New York Turtle and Tortoise Society, Nueva York, Estados Unidos.

Congdon, J.D., \& J.W. Gibbons. 1985. Egg components and reproductive characteristics of turtles: Relationships to body size. Herpetologica 41: 194-205.

Congdon, J.D. \& J.W. Gibbons. 1990. Turtle eggs: Their ecology and evolution, pp. 109-123. In J.W. Gibbons (ed.). Life history and ecology of the slider turtle. Smithsonian Institution, Washington D.C.

Dunham, A.E., P.J. Morin \& H.M. Wilbur. 1988. Methods for the study of reptile populations, pp. 331-386. In C. Gans \& R. Huey (eds.). Biology of the Reptilia. Vol. 16: Defense and life history. Liss, Nueva York.

Ernst, C.H. \& R.W. Barbour. 1989. Turtles of the World. Smithsonian Institution. Estados Unidos. pp 203-210.

Ewert, M.A. 1979. The embryo and its egg: Development and natural history, pp. 333-413. In M. Harless \& H. Morlock (eds.). Turtles: Perspectives and Research. Wiley. Nueva York.

Fachin, A. 1994. Depredación de la Taricaya, Podocnemis unifilis, en la Reseva Nacional Pacaya- Samiria, Loreto, Perú. Bol. Lima 15(91-96): 417-423.

Frazier, J.G. 1997. Management of tropicals chelonians: ¿Dream or nightmare? Part 2. In J. Van Abbema (ed.). Proceedings: Conservation, Restoration and Management of Tortoises and Turtles - An International Conference. New York Turtle and Tortoise Society, Nueva York, Estados Unidos. Garduño-Andrade, M. \& E.
Cervantes. 1996. Influencia de la temperatura y la humedad en la sobrevivencia en nidos in situ y en corral de tortuga carey (Eretmochelys imbricata) en las Coloradas, Yucatán, México. Cien. Pesq. 12: 90.

Gibbons, J.W. 1990.Turtle studies at SREL: A Research perspective, pp. 19-44. In J.W. Gibbons (ed.). Life History and Ecology of the Slider Turtle. Smithsonian Institution, Washington, D.C.

Groombridge B. 1982. The IUCN Amphibiam-Reptilia Red Data Boock. Gland, Suiza.

Medem, F. 1962. La distribución geográfica y ecología de los Crocodylia y Testudinata en el departamento del Chocó. Rev. Acad. Colomb. Cien. Fís. Natur. 11 (14): 279-303.

Medem, F. 1975. La reproducción de la icotea, Pseudemys scripta callirostris (Testudines, Emydidae). Caldasia 11: 83-106.

Moll, E.O. \& J.M. Legler. 1971. The life history of a neotropical slider turtle, Pseudemys scripta (Schoepff), in Panamá. Bull. Los Angeles Co. Mus. Natur. Hist. Sci. 11: 1-102.

Moll, D. 1986. The distribution, status, and level of exploitation of the freshwater turtle Dermatemys mawei in Belize, Central America. Biol. Conserv. 35: 87-96.

Morris, K.A., G.C. Packard, T.J. Boardman, G.L., Paukstis \& M.J. Packard. 1983. Effect of the hydric environment on growth of embryonic snapping turtles (Chelydra serpentina). Herpetologica 39: 272-285.

Pritchard, P.C \& P. Trebbau. 1984. The Turtles of Venezuela. Contributions to Herpetology 2. Society for the Study for Amphibians and Reptiles.

Rand, A.S. \& M.H. Robinson. 1969. Predation on iguana nest. Herpetologica 25: 172-174

Semlitsch, R.D. \& J.W. Gibbons. 1978. Reproductive allocation in the brown water snake Natrix taxispilota. Copeia 1978: 721-723.

Stewart, J.R. 1979. The balance between number and size of young in the live bearing lizard Gerrhotus coeruleus. Herpetologica 35: 342-350.

Temple, S.A. 1987. Predation on turtle nests increases near ecological edges. Copeia 1987: 250-252.

Wilbur, H.M. \& P.J. Morin. 1988. Life history evolution in turtles, pp. 387-439. In C. Gans \& R. Huey (eds.). Biology of the Reptilia. Vol. 16: Defense and life history. Liss, Nueva York. 